\title{
A VLP for validation of the Plasmodium falciparum circumsporozoite protein junctional epitope for vaccine development
}

\author{
Erwan Atcheson ${ }^{1}$, Adrian V. S. Hill ${ }^{1}$ and Arturo Reyes-Sandoval $\mathbb{i D}^{1,2}$
}

Malaria continues to be a pressing global health issue, causing nearly half a million deaths per year. An effective malaria vaccine could radically improve our ability to control and eliminate this pathogen. The most advanced malaria vaccine, RTS,S, confers only $30 \%$ protective efficacy under field conditions, and hence the search continues for improved vaccines. New antigens and formulations are always first developed at a pre-clinical level. This paper describes the development of a platform to supplement existing tools of pre-clinical malaria vaccine development, by displaying linear peptides on a virus-like particle (VLP). Peptides from PfCSP, particularly from outside the normal target of neutralizing antibodies, the central NANP repeat region, are screened for evidence of protective efficacy. One peptide, recently identified as a target of potent neutralizing antibodies and lying at the junction between the $\mathrm{N}$-terminal domain and the central repeat region of PfCSP, is found to confer protective efficacy against malaria sporozoite challenge in mice when presented on the Q $\beta$ VLP. The platform is also used to explore the effects of increasing numbers of NANP unit repeats, and including a universal $C D 4^{+}$T-cell epitope from tetanus toxin, on immunogenicity and protective efficacy. The VLP-peptide platform is shown to be of use in screening malaria peptides for protective efficacy and answering basic vaccinology questions in a pre-clinical setting.

npj Vaccines (2021)6:46; https://doi.org/10.1038/s41541-021-00302-x

\section{INTRODUCTION}

The need for an effective malaria vaccine is pressing, with almost half a million deaths and 148-304 million clinical cases every year caused by this parasite ${ }^{1}$. The leading malaria vaccine, RTS,S represents a major milestone in malaria vaccine development. However, it is only about 30\% efficacious under field conditions ${ }^{2}$, which (at \$5-10 per dose) will make it less cost-effective at preventing severe malaria than long-lasting insecticide-treated bednets ${ }^{3}$.

A peptide-based vaccine was amongst the first malaria vaccines to reach clinical study ${ }^{4}$. It consisted of three copies of the NANP tetramer motif from the central repeat region of the major malaria sporozoite antigen, circumsporozoite protein (CSP). This region, and the NANP motif, in particular, had been shown to be a target of neutralising antibodies ${ }^{5,6}$. Despite showing some protective efficacy in a clinical trial, however, it became clear that a construct fusing the repeat region (R) to the Hepatitis B surface antigen (S) was much more immunogenic ${ }^{7}$. When the C-terminal domain of PfCSP, containing a helper T-cell epitope, was included $(T)$, this became the RTS,S vaccine ${ }^{8}$, the only malaria vaccine, or indeed parasite vaccine, to reach Phase III clinical trials ${ }^{2}$. RTS, $S$ is currently undergoing limited pilot distribution in Ghana, Kenya, and Malawi (WHO press release, 24 April 2017).

Although the immunogenicity of short peptides is enhanced by coupling them to virus-like particles (VLPs) ${ }^{9}$, the aim of this paper is not necessarily to advocate VLP-peptide vaccines for clinical use, nor is the aim to obtain high levels of protective efficacy against challenge; one of the main metrics of vaccine efficacy used here is a delay in time to reach $1 \%$ blood-stage parasitaemia, previously validated $^{10,11}$ and in fact a more sensitive metric to use when testing the hypothesis that combining vaccines enhances efficacy since sterile protection represents saturation ${ }^{12}$. Rather, the focus of this paper is to explore the potential of VLP-peptide vaccines in pre-clinical screening of epitopes for potential protective efficacy and to answer other basic questions useful to malaria vaccine development. This platform, chemically coupling synthetic peptides via a terminal cysteine residue to the $Q \beta$ VLP derived from the $Q \beta$ bacteriophage ${ }^{13}$, is tractable and easy to use, allowing novel vaccines to be quickly and inexpensively produced for use in murine malaria vaccine studies.

The utility of this platform in achieving these ends is here demonstrated by the discovery of a linear peptide derived from PfCSP which, when coupled to the VLP Q $\beta$, generates neutralising antibodies capable of protecting mice against challenge with transgenic malaria parasites encoding $P$. falciparum CSP (PfCSP). This finding is given greater significance by the recent identification of neutralising monoclonal antibodies, generated by individuals receiving a radiation attenuated sporozoite vaccine, which recognise this sequence ${ }^{14,15}$; but previous attempts to generate such antibodies in mice by vaccination have not succeeded until now. This peptide sequence lies between the central repeat region and the N-terminal domain of PfCSP and is not included in the $\mathrm{RTS}, \mathrm{S}$ vaccine.

The $Q \beta$-peptide platform is also used to screen linear epitopes recognised by another recently identified neutralising antibody ${ }^{16}$, spanning the highly conserved "Region I" sequence of the $\mathrm{N}$ terminal domain of PfCSP. These peptides, coupled to $\mathrm{Q} \beta$, are highly immunogenic and the antibodies so generated can recognise native CSP; but they confer no protective efficacy on vaccinated mice. This is of use in demonstrating that more needs done to exploit this epitope in a successful vaccination strategy.

\footnotetext{
${ }^{1}$ The Jenner Institute, University of Oxford, Oxford, UK. ${ }^{2}$ Instituto Politécnico Nacional, IPN. Av. Luis Enrique Erro s/n. Unidad Adolfo López Mateos, Mexico City, Mexico.

更email: arturo.reyes@ndm.ox.ac.uk
} 
Finally, the $Q \beta$-peptide platform is used to determine the effects of increasing the number of NANP repeats per peptide, and of fusing a $\mathrm{CD}_{4}^{+}$T-cell epitope from tetanus toxin to a NANP repeat peptide. Increasing the number of NANP repeats improves immunogenicity and protective efficacy, and including the tetanus, toxin epitope improves anti-NANP antibody affinity, which is shown to be an important correlate of protection.

The discoveries reported here, made using the $\mathrm{Q} \beta$-peptide platform, will be of utility in informing strategies to improve the protective efficacy of the RTS,S vaccine or successors.

\section{RESULTS \\ Immunogenicity and protective efficacy of short PfCSP peptides presented on $\mathbf{Q} \boldsymbol{\beta}$}

When coupled to $Q \beta$, a peptide representing three repeats of the NANP sequence from the central repeat region of PfCSP generates high-titre antibodies (Fig. 1A) and partially protects $\mathrm{BALB} / \mathrm{c}$ mice from challenge with transgenic PfCSP Plasmodium berghei sporozoites (Fig. 1B).

To see if peptides from other regions of PfCSP could likewise generate protective immunity when administered as Q $\beta$-peptide vaccines, two peptides ("RI short" and "RI long") was constructed based on the core and expanded linear sequence recognised by a neutralising monoclonal that targets the $\mathrm{N}$-terminal domain of PfCSP in Region I; see Fig. 2A. Despite being highly immunogenic in homologous and heterologous peptide enzyme-linked immunosorbent assays (ELISAs) (Fig. 2B, C), and, in the case of RI-long, showing cross-species reactivity against full-length PvCSP-210 (Fig. 2D), neither of these Region I-based $\mathrm{Q} \beta$-peptide vaccines conferred any protection against challenge with transgenic PfCSP $P$. berghei sporozoites (Fig. $2 \mathrm{H}$ ). In addition, a $\mathrm{Q} \beta-(\mathrm{NVDP})_{6}$ vaccine was tested, to see if antibodies against the NVDP tetramer, present in three non-consecutive copies in the PfCSP 3D7 sequence, could neutralise sporozoites, given the protective efficacy of anti-NANP antibodies. Despite generating antibodies against (NVDP) ${ }_{6}$ and a native peptide from PfCSP containing a copy of NVDP ("ADGN long") (Fig. 2E), the $\mathrm{Q} \beta-(\mathrm{NVDP})_{6}$ vaccine showed no protective efficacy (Fig. $2 \mathrm{H}$ ). The lack of efficacy of these three vaccines demonstrates that any contribution to protective efficacy from the adjuvant, Matrix $M$, alone, is absent or negligible.

A forth peptide from PfCSP, "ADGN long", lying adjacent to Region $\mathrm{I}$ at the junction between the $\mathrm{N}$-terminal domain and the repeat region of PfCSP, (see Fig. 2A for sequence and position) was likewise chemically coupled to $\mathrm{Q} \beta$ and administered to mice.
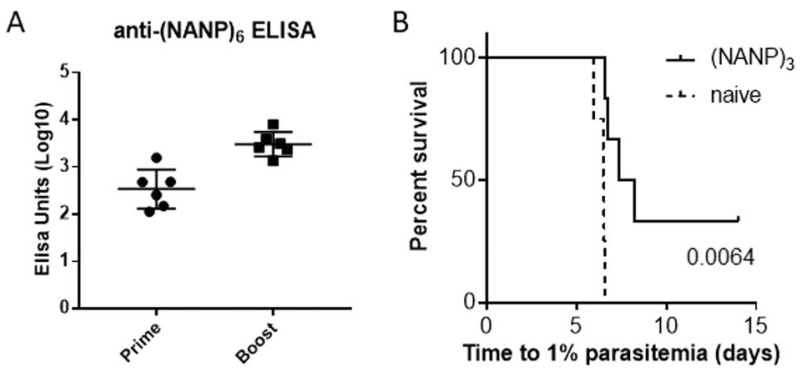

Fig. 1 Immunogenicity and protective efficacy of a Q $\beta$-(NANP) 3 vaccine. $\mathrm{BALB} / \mathrm{c}$ mice $(n=6)$ per group were vaccinated by intramuscular injection $(20 \mu \mathrm{g}$ per dose, two shots, 3-week primeboost interval) with (NANP) ${ }_{3}$ chemically coupled to $\mathrm{Q} \beta$ virus-like particle and delivered with Matrix- $\mathrm{M}^{\mathrm{TM}}$ adjuvant. A Standard curve ELISAs performed to determine the antibody response against $(\mathrm{NANP})_{6}$ peptide. Means are shown \pm SD. B Mice were challenged by intravenous injection of 1000 PfCSP-replacement $P$. berghei sporozoites. The time to reach $1 \%$ blood-stage parasitaemia was calculated by linear regression from daily thin blood smears. $P$ values from Log-rank tests in comparison to naïves are shown.
$Q \beta-(A D G N$ long) was found to be both highly immunogenic (Fig. 2F, G) and partially protected mice against challenge with transgenic PfCSP P. berghei sporozoites (Fig. 2H).

$Q \beta$-(ADGN long) contains a single NANP tetramer within its sequence, and generates anti-NANP antibodies (Fig. 2F). These are, however, of extremely low affinity (Fig. 2G). This suggested that these anti-NANP antibodies were not principally responsible for mediating the protective efficacy of the $Q \beta$-(ADGN long) vaccine.

In theory, two vaccines generating immune responses against different targets will, if either is weakly protective alone, show markedly enhanced efficacy when combined ${ }^{12}$. To test whether "ADGN long" represents a truly novel epitope, rather than its effects being mediated through anti-NANP antibodies as with $\mathrm{Q} \beta$ $(\mathrm{NANP})_{6}$, an experiment was carried out combining $Q \beta-(A D G N$ long) and $\mathrm{Q} \beta$-(NANP) ${ }_{6}$ vaccines under suboptimal conditions, with dosage reduced compared to previous experiments (Fig. 1) to allow maximum sensitivity in detecting any enhancement in protective efficacy. Enhanced protective efficacy was obtained with the combination of $\mathrm{Q} \beta-(\mathrm{NANP})_{6}$ and $\mathrm{Q} \beta-(\mathrm{ADGN}$ long) compared with either subunit vaccine alone (Fig. 3B). Given that the anti-(NANP) 6 and anti-(ADGN long) titres and avidity indices were the same in the single-component and combination groups (Fig. 3A), the only remaining explanation for enhanced efficacy is that these vaccines elicit immune responses against different epitopes.

To further test whether the enhancement in protective efficacy was consistent with theoretical predictions, a mathematical model developed by our group ${ }^{12}$ was adapted to present purposes. From that mathematical model, the following expression can be derived

$\frac{L_{V}}{L_{N}}=i_{V 1} * i_{V 2}=g^{T n-T_{V}}$

where $L_{V}$ and $L_{N}$ are the numbers of infected hepatocytes in vaccinated and naïve mice, respectively; $i_{V 1}$ and $i_{V 2}$ are the reductions in infectivity caused by two different immune components (in this case, against (NANP) 6 and ADGN-long); $g$ is the blood-stage growth rate (calculated in ref. ${ }^{12}$ to be $g=3.5$ ); and $T_{N}$ and $T_{V}$ are the time-to- $1 \%$ values of the naïve and vaccinated mice, respectively. The reduction in liver load for each group compared to naïve mice can be calculated as $100^{*}(1-$ $\left.i_{V 1}{ }^{*} i_{V 2}\right)$ and is shown graphically in Fig. $3 C$, where median values are used for the calculations as the distribution of $T$ is theoretically skewed $^{12}$; this can be seen in the present data where the time-to$1 \%$ values in challenged naïve mice fall in a normal range of 5.6-6.4 d (Fig. 3B) but with the two mice showing time-to-1\% of $6.4 \mathrm{~d}$ showing extrapolated liver burdens $50 \%$ less than the four mice showing time-to- $1 \%$ of $5.6 \mathrm{~d}$. The time-to- $1 \%$ was also half a day longer than in the previous experiment (Fig. 2) explaining the lack of efficacy of Q $\beta$-(NANP) 6 alone (Fig. 3). A predicted value for the reduction in liver load in the combination NANP + ADGN group can be calculated from the actual values of the $\mathrm{Q} \beta$-(NANP) 6 and $\mathrm{Q} \beta$-(ADGN long)-only groups, which have reductions in a liver load of $54 \%$ and $57 \%$, respectively. The predicted reduction in liver load from the combination NANP + ADGN group is $80 \%$, and the actual reduction is $89 \%$. A Wilcoxon signed-rank test comparing the predicted and actual reduction confirms the values to be very close, with a $p$ value for discrepancy of 0.44 .

In summary, the $\mathrm{Q} \beta$-peptide platform enabled the discovery of a neutralising PfCSP epitope. The utility of the platform was further demonstrated by investigating the effect of increasing the number of (NANP) units per peptide.

Increasing the number of units repeats in Q $\beta$-(NANP) vaccines Peptides consisting of 1, 2, 3 or 6 NANP repeats were synthesised and coupled to $\mathrm{Q} \beta$ to see if increasing the number of NANP repeats would increase the immunogenicity of $Q \beta$-(NANP) vaccines. ELISAs performed on sera from $B A L B / c$ mice vaccinated 
A

\begin{tabular}{|l|l|}
\hline (NANP) $_{6}$ & NANPNANPNANPNANPNANPNANP \\
\hline Rl short & EDNEKLRKPKH \\
\hline Rl long & DDGNNEDNEKLRKPKHKKLKQPADGN \\
\hline ADGN long & ADGNPDPNANPNVDP \\
\hline
\end{tabular}
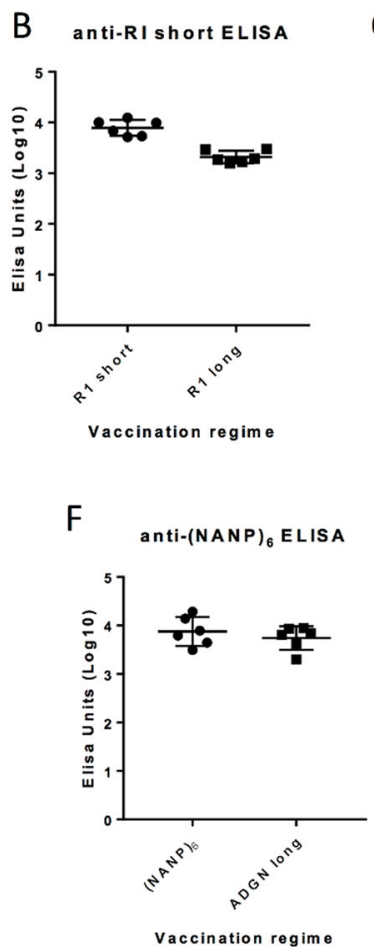

Vaccination regime

$\mathrm{F}$

Vaccination regime
C

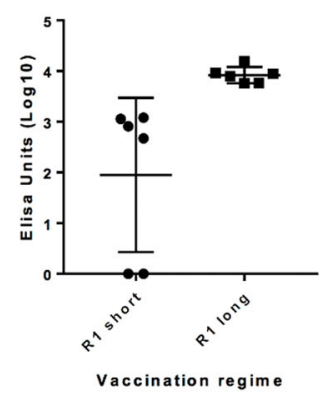

G

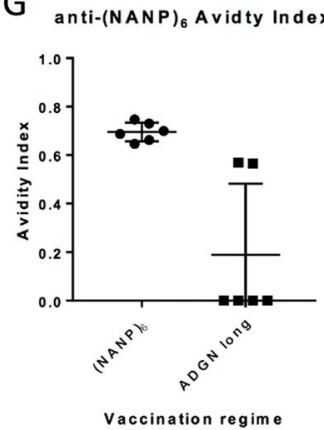

D

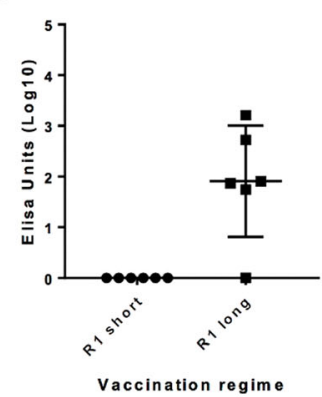

Vaccination regime

>gi|15375371 | emb |CAB38998.2 | circumsporozoite (CS) protein [Plasmodium falciparum 3D7] 作 EKLRKPKHKKLKOPADGNPDPNANPNVDPNANPNVDPNANPNVDPNANPNANPNANPNANPNANPNANPNANPNANPN ANPNANPNANPNANPNANPNANPNANPNANPNANPNVDPNANPNANPNANPNANPNANPNANPNANPNANPNANPN ANPNANPNANPNANPNANPNANPNANPNANPNANPNKNNQGNGQGHNMPNDPNRNVDENANANSAVKNNNNEEPS DKHIKEYLNKIQNSLSTEWSPCSVTCGNGIQVRIKPGSANKPKDELDYANDIEKKICKMEKCSSVFNVVNSSIGLIMVLSFLFLN

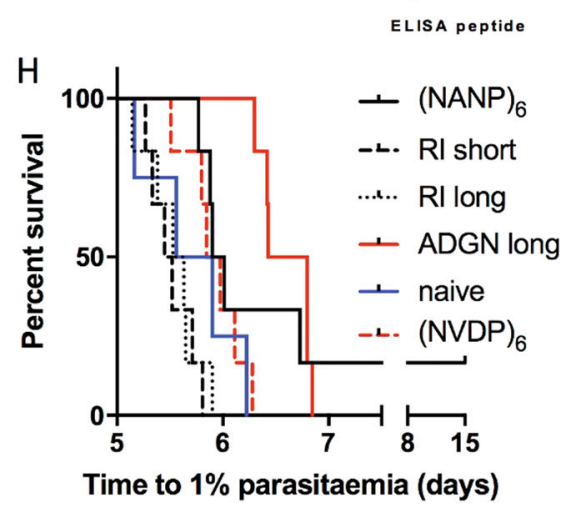

E $Q \&(N V D P)_{6}$ vaccination

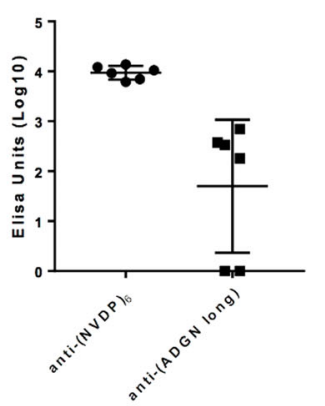

ELISA peptide

Fig. 2 Immunogenicity and protective efficacy of peptides derived from PfCSP delivered as Q $\beta$-peptide vaccines. BALB/c mice $(n=5-6$ per group) were vaccinated with non-repeat region CSP peptides chemically coupled to Q $\beta$ ( $3 \mu$ g per dose, by intramuscular injection, delivered with Matrix- $M^{\mathrm{TM}}$ adjuvant, three shots using 3-week intervals.) A $P$. falciparum CSP peptides used as Q $\beta$-peptide vaccines and location in PfCSP sequence. Standard curve ELISAs were performed using sera taken 2 weeks after the final vaccination, against B "RI short" peptide, C "RI long" peptide, D full-length PvCSP, or F (NANP) 6 peptide; E shows mice vaccinated with Q $\beta$-(NVDP) 6 and ELISAs against peptides shown on the $x$-axis. G Avidity index represents the ratio of sera treated with $7 \mathrm{M}$ urea to untreated sera in ELISAs. Means are shown \pm SD. Vaccination received shown on $x$-axis except in the case of $(\mathbf{E})$. $\mathbf{H}$ Mice were challenged 3 weeks after the final shot with 1000 transgenic $P$. berghei sporozoites expressing PfCSP, and time to reach $1 \%$ blood-stage parasitaemia determined by linear regression using daily thin blood smears.

with these $\mathrm{Q} \beta$-(NANP) ${ }_{n}$ vaccines demonstrated an apparently linear relationship between titre and number of NANP repeats (Fig. 4A), at least for the first two shots (first shot, $r^{2}=0.986, p=$ 0.007 ; second shot, $\left.r^{2}=0.992, p=0.0039\right)$. The relation still existed, though more loosely, after a third vaccination $\left(r^{2}=\right.$ $0.890, p=0.0028$ ) but a failure to boost titres in most mice after a fourth shot seems to have terminated the relationship $\left(r^{2}=0.815\right.$, $p=0.097)$. Excluding the non-boosting fourth shot, the relationship between a number of shots and titre also appears to be linear for $\mathrm{Q} \beta$-(NANP) $)_{2}\left(r^{2}=0.394, p=0.005\right), \mathrm{Q} \beta$-(NANP) $)_{3}\left(r^{2}=0.916, p<\right.$ $0.0001)$ and $\mathrm{Q} \beta$-(NANP) $6\left(r^{2}=0.643, p<0.0001\right)$, though the linearity of this relationship is perhaps only convincing for $Q \beta$ $(\mathrm{NANP})_{3}$ (Fig. 4B). The avidity index was also highest for mice vaccinated with $\mathrm{Q} \beta$-(NANP) 6 (Fig. $4 \mathrm{C}$ ), and higher titres and affinity translated into increasing protection with increasing numbers of NANP repeats (Fig. 4D).

$\mathrm{Q} \beta$-(NANP) $)_{1}$ - and $\mathrm{Q} \beta-(\mathrm{GG})_{4}(\mathrm{NANP})_{1}$-vaccinated mice, though generating no or low responses against $(N A N P)_{6}$ in ELISAs, did generate high titres against (NANP) $)_{1}$ and $(\mathrm{GG})_{4}(\mathrm{NANP})_{1}$ peptide as ELISA coating antigens; indeed, they generated higher titres against those peptides than any $\mathrm{Q} \beta$-(NANP) $n$ with two or more NANP repeats (Fig. 4E). Similarly, $Q \beta$-(NANP) ${ }_{2}$-vaccination generated the highest titres in an anti-(NANP) ${ }_{2}$ ELISA. In anti-(NANP) $)_{3}$ ELISAs $\mathrm{Q} \beta$-(NANP) 6 mice produced the highest titres, as with
$(\mathrm{NANP})_{6}$ ELISAs, suggesting that (NANP) $n$ peptides of one or two repeats have a different conformation from that of three or more.

Theoretically, the relationship between the number of NANP repeats and anti-(NANP) 6 titre is not expected to remain linear indefinitely. The point at which the relationship saturates is yet to be experimentally determined, but the mathematical model developed below suggests that it will saturate at some point. The model, relating titre to dose, $V$, a number of unit repeats, $N$, and density of peptides, $\partial$, on each VLP, derives from the following assumptions:

1. There is a fixed and finite quantity of epitope-specific B-cells in a naïve mouse, equal to $B_{\text {max }}$.

2. The net quantity of antibodies $R$ secreted by a given activated epitope-specific B-cell $B_{A}$ and descendent B-cells resulting from its proliferation will be a constant: $R=f^{*} B_{A}$, where $f$ is a function describing the number of B-cells generated by the proliferation of the original activated B-cell $B_{A}$ and the number of antibodies released by each.

3. The probability that a given epitope on a given VLP binds and activates a B-cell after a VLP/B-cell encounter, $p_{n}$, is independent of $\partial, V$, and $N$.

4. The probability $p_{a}$ that a given B-cell is activated is independent of the activation status of any other B-cells. 


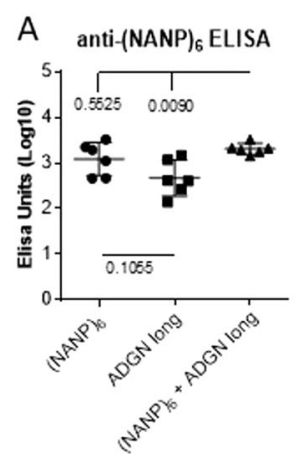

B

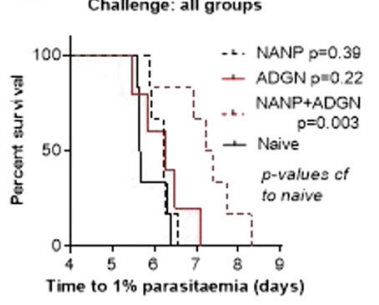

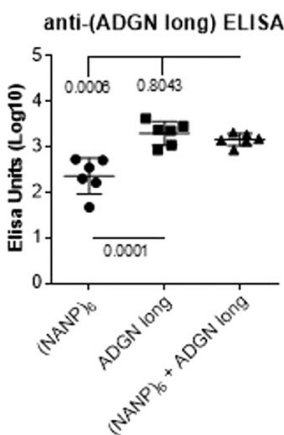

Challenge: $A D G N$ vs NANP+ADGN

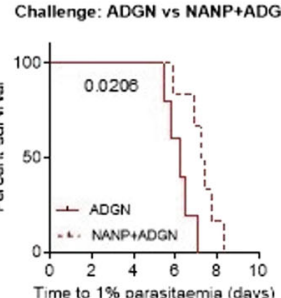

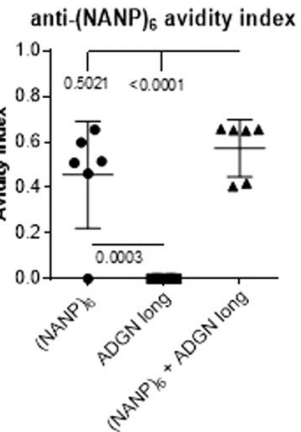
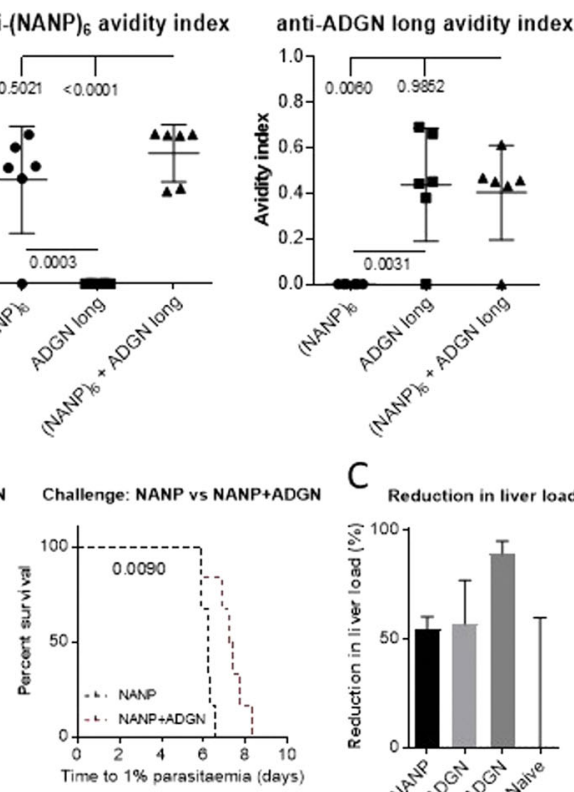

C

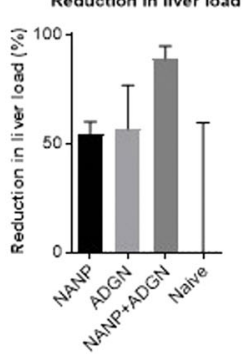

Fig. 3 Effects on immunogenicity and protective efficacy of combining $Q \beta$-(NANP) ${ }_{6}$ and $Q \beta$-(ADGN long) vaccines. BALB/c mice $(n=6$ per group) were vaccinated with two shots by intramuscular injection of $1 \mu \mathrm{g}$ of each $\mathrm{Q} \beta$-peptide in Matrix-M $\mathrm{M}^{\mathrm{TM}}$ adjuvant into separate legs using a 3-week prime-boost interval. A Standard curve ELISAs from sera taken 2 weeks post-boost, and avidity. Numbers represent $p$ values from ANOVA with Bonferroni's multiple comparisons test. B Mice were challenged 3 weeks post-boost by intravenous injection of 1000 PfCSP replacement $P$. berghei sporozoites into the tail vein. The time to reach $1 \%$ blood-stage parasitaemia was calculated by linear regression from daily thin blood smears. Numbers represent $p$-values from log-rank tests between indicated groups. Means are shown \pm SD. C Reduction in liver load, the ratio of infected hepatocytes in vaccinated $\left(L_{V}\right)$ compared to naïve mice $\left(L_{N}\right)$, was calculated using the formula Reduction (\%) $=$ $\left(1-i_{V}\right)^{*} 100$, where the vaccine-reduced infectivity $i_{V}=L_{V} / L_{N}=g^{T n-T_{V}}$, where $g$ is the blood-stage growth rate, $T_{N}$ the median time-to-1\% in naïve mice, and $T_{V}$ the time-to- $1 \%$ in vaccinated mice.

If $\partial, V$ and $N$ are given, then the number of activated B-cells $B_{A}$ is

$B_{A}=B_{\max } * p_{a}$

Since $R=f * B_{A}$, the maximum number of antibodies released $R_{\max }=f^{*} B_{\text {max }}$. Therefore

$R=R_{\max } * p_{a}$

Failure to activate a B-cell, $p_{-a}$, occurs when each unit epitope on each VLP which encounters a B-cell fails to trigger activation. A given unit epitope on a VLP has a probability $p_{n}$ of activating the Bcell, and probability $p_{-n}=1-p_{n}$ of failing to activate the B-cell. On a given VLP there are $\partial^{*} N$ unit epitopes; the probability that they all fail to activate the B-cell is $\left(1-p_{n}\right)^{\partial^{*} N}$. If $p_{V}$ is the probability that a VLP encounters a B-cell, then on average a B-cell will have $p_{V}{ }^{*} V$ encounters. Thus the probability that each unit epitope on each VLP which encounters a B-cell fails to activate it is $p_{-a}=\left(1-p_{n}\right)^{\partial^{*} N^{*} p V^{*} V}$. If the dose $V$ is expressed in arbitrary units (e.g. $\mu$ g or ng) then $p_{V}$ can for practical purposes be set equal to one. Thus

$p_{a}=1-p_{-a}=1-\left(1-p_{n}\right)^{\partial * N * V}$

and from (3) and (4)

$R=R_{\max } *\left(1-\left(1-p_{n}\right)^{\partial * N * V}\right)$

Equation (5) suggests that the relationship will only be linear at small scales, and should in fact be saturating. This is found to be the case with an experiment in mice comparing two Hepatitis B surface antigen-based VLPs, R21, and RTS,S, which differ only in the density of CSP antigen per VLP; differences in the immune profile between the two VLPs fit very closely the predicted difference from the model above (Adrian Hill, pers. comm).
Thus, increasing the number of NANP tetramers per peptide improves immunogenicity and protective efficacy, though in theory with diminishing returns. Next, the platform was used to investigate whether including a short $\mathrm{CD}^{+}{ }^{+} \mathrm{T}$-cell epitope from tetanus toxin would improve the affinity and protective efficacy of $\mathrm{Q} \beta$-(NANP)-based vaccines.

\section{Effects on immunogenicity and protective efficacy of fusing a} tetanus toxin $\mathrm{CD}^{+}{ }^{+}$T-cell epitope with (NANP) 3

Despite similar anti-(NANP) 6 titres (Fig. 5A), mice vaccinated with $\mathrm{Q} \beta$-(NANP) ${ }_{6}$ show variable outcomes following challenge (Fig. 5B). When titres are very similar, the affinity of anti-(NANP) ${ }_{6}$ antibodies associates strongly with the outcome, when mice are divided into two groups (protected or showing delay in time to reach $1 \%$ parasitaemia, vs. no evidence of delay) or three groups, as shown in Fig. 5C.

For this reason, the P2 universal T-cell epitope from tetanus toxin (tt) was fused to (NANP) $)_{3}$ to see if this would improve the affinity and hence the protective efficacy of (NANP)-based vaccines. Figure $6 \mathrm{~A}$ shows a marginal improvement in titre $(p=0.56$, Fig. $6 \mathrm{~A})$, but a highly significant increase in the avidity index of anti-(NANP) ${ }_{6}$ antibody $(p=0.0029$, Fig. 6B) resulting from the inclusion of the tt epitope with (NANP) ${ }_{3}$, compared to $(\mathrm{NANP})_{3}$ alone, presented on $\mathrm{Q} \beta$. Improved protective efficacy was seen with $\mathrm{Q} \beta$-tt-(NANP) ${ }_{3}$ vaccination compared with $\mathrm{Q} \beta$ $(\mathrm{NANP})_{3}$, but this was not significant $(p=0.1$, Fig. $6 \mathrm{C})$. Thus, adding a universal $\mathrm{CD}^{+}$epitope to a NANP-based vaccine can improve antibody affinity, and thus potentially increase protective efficacy. 
A

Anti-(NANP) 6 ELISA 1st shot
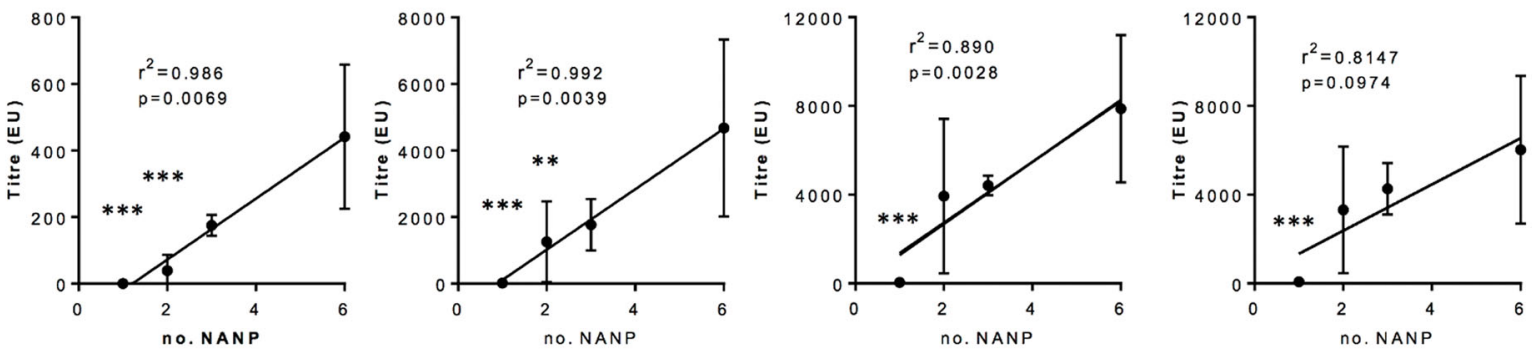

B

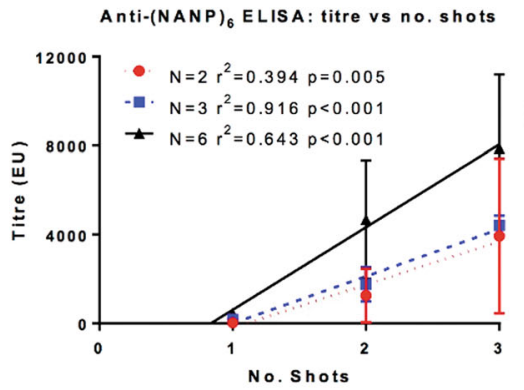

c

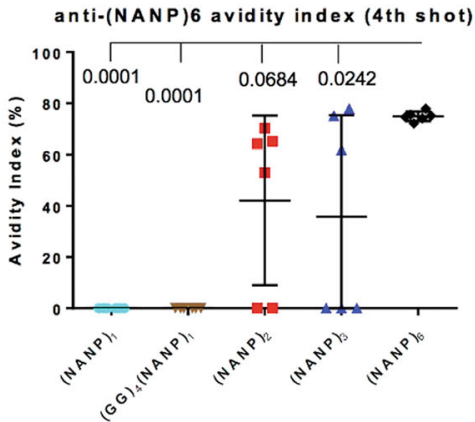

D

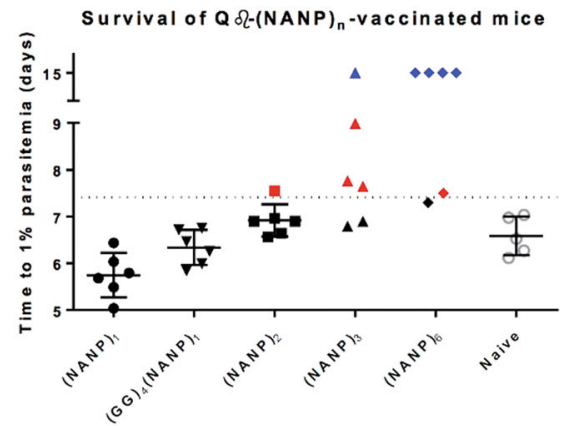

E

anti-(NANP) ${ }_{1}$ ELISA

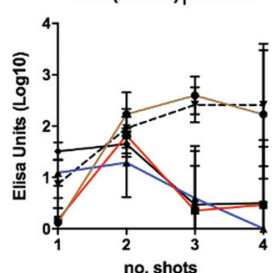

anti-(GG) ${ }_{4}(\mathrm{NANP})_{1}$ ELISA

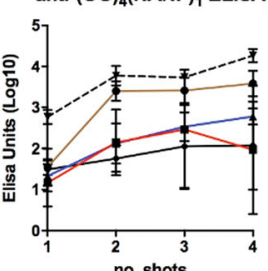

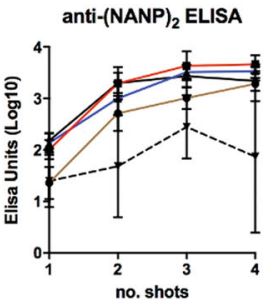
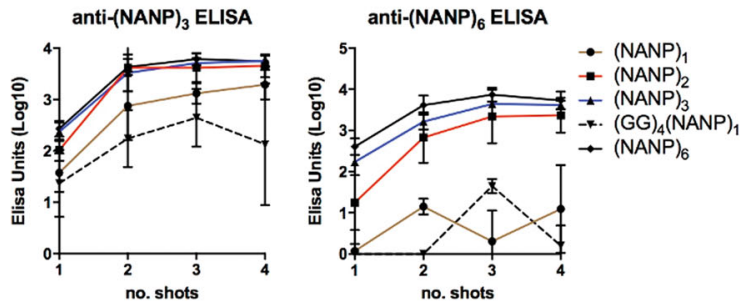

Fig. 4 Effects on immunogenicity and protective efficacy of increasing the number of units repeats in Q $\beta$-(NANP) vaccines. Q $\beta$-(NANP) VLPs were constructed by synthesising peptides with varying numbers of (NANP) unit repeats (no. NANP $=1,2,3,6)$ and $(G G)_{4}(N A N P)_{1}$ and chemically coupling them to $\mathrm{Q} \beta$. These VLPs were used to vaccinate BALB/c mice ( $n=6$ per group) by intramuscular injection of $3 \mu \mathrm{g}$ doses in Matrix-M $M^{T M}$ adjuvant, with four shots given at 3-week intervals. A Serum was taken 2 weeks after each shot and used in standard curve ELISAs against (NANP) $6 . r^{2}$ and $p$ values from linear regressions are shown. Asterix represents significance levels of $p<0.01(* *)$ and $p<0.001(* * *)$ by ANOVA in comparison to Q $\beta$-(NANP) 6 . B Titre versus a number of shots for each Qb-(NANP) $)_{n}$ group, with $N=2, N=3$ or $N=6$ as shown. C The avidity indices 2 weeks post-fourth shot; shown are $p$ values from ANOVA with Bonferroni's multiple comparisons test in comparison to Q $\beta$-(NANP) ${ }_{6}$-vaccinated mice. D Three weeks after the fourth vaccination mice were challenged by intravenous injection of 1000 PfCSP transgenic $P$. berghei sporozoites. Thin blood smears were taken daily to determine the time to reach $1 \%$ blood-stage parasitaemia by linear regression. In challenge data, points coloured blue represent sterile protection, and points coloured red to represent a delay in time-to-1\%, defined as time-to- $1 \%$ greater than average naïve time-to-1\% plus 2 standard deviations, with the boundary represented by a dotted line. E Sera from mice in (A) were also used to determine antibody titres specific for each variable number of (NANP) repeats, 2 weeks post-vaccination; graph titles represent the peptide used to coat ELISA plates, and lines representing vaccination groups as shown. Means are shown \pm SD.

\section{DISCUSSION}

The VLP-peptide platform is a simple and straightforward way of answering important questions in vaccine development, having been used here to identify a target of neutralising antibodies in PfCSP, establish that increasing numbers of the NANP unit repeat improves immunogenicity and protective efficacy, and that inclusion of a CD4 ${ }^{+}$T-cell epitope can improve the affinity of anti-(NANP) antibodies.

The finding that protective immunity against malaria can be generated by $Q \beta-(A D G N$ long) vaccination of mice is particularly exciting, as this junctional epitope, situated between $\mathrm{Rl}$ in the $\mathrm{N}$ terminal domain and the central repeat region of PfCSP, has been recently identified as a target of potently neutralising dualspecificity antibodies generated in humans following irradiated sporozoite (PfSPZ) vaccination ${ }^{14,15}$. Such antibodies recognise both the junctional epitope and the central repeat region;
$Q \beta$-(ADGN long) also elicits antibodies against both regions, and it would be interesting to find out if these antibodies are likewise of dual specificity. Vaccination of BALB/c mice by conjugation of KQPADGNPDPNANPNVDPN to keyhole limpit haemocyanin (KLH) carrier protein elicited only non-neutralising antibodies ${ }^{15}$. The success of our vaccination regime is likely due to the use of a VLP, $\mathrm{Q} \beta$, to present the junctional peptide, as VLPs elicit higher titres of the antibody with higher affinity. If this is the reason for the difference in outcomes, it underscores the utility of the VLPpeptide platform to screen epitopes for protective efficacy, as carrier proteins such as $\mathrm{KLH}$, although inducing antibody responses to peptides, are probably not sufficiently immunogenic to raise high-quality, high-titre antibodies. However, it is possible that the addition of 'KQP' at the beginning of the above sequence abrogates the production of neutralising antibodies. It will be important to settle this question, as the most obvious application 
A

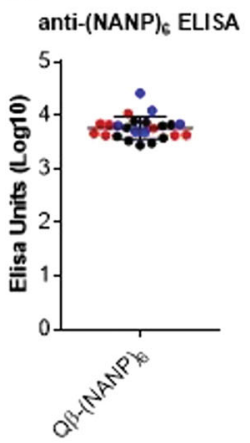

B

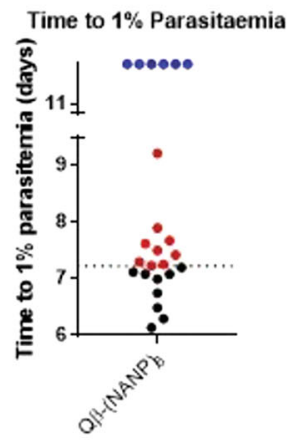

C

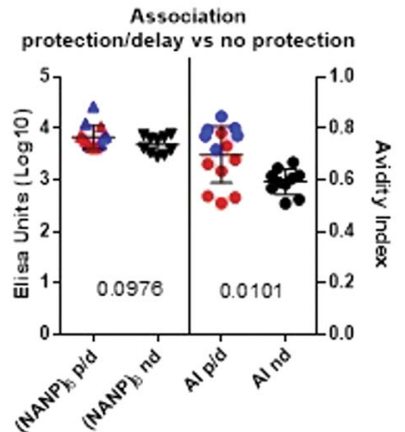

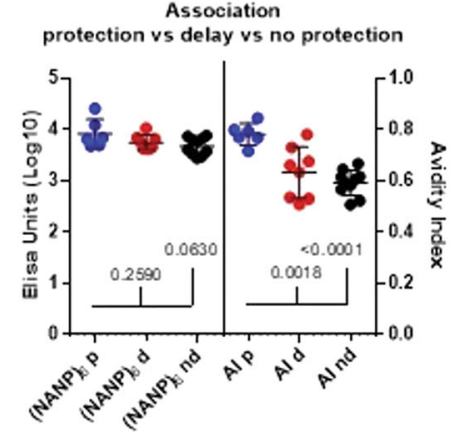

Fig. 5 Avidity index as a correlate of protection in Q $\beta$-(NANP) $)_{6}$-vaccinated mice. BALB/c mice $(n=24)$ were vaccinated with $Q \beta-(N A N P)_{6}$ by intramuscular injection, using $1 \mu \mathrm{g}$ per dose, two shots, a 3-week prime-boost interval, and Matrix-M ${ }^{\mathrm{T} M}$ adjuvant. A Serum was collected from mice 2 weeks post-boost and standard curve ELISA performed against (NANP) 6 . B Mice were challenged intravenously with 1000 PfCSP transgenic $P$. berghei sporozoites 3 weeks post-boost. Daily thin blood smears were used to determine the time to reach $1 \%$ blood-stage parasitaemia by linear regression. Mice with sterile protection are coloured blue, and those with a delay in time to reach $1 \%$ blood-stage parasitaemia coloured red, where "delay" is defined as time-to- $1 \%$ greater than the mean of naïve challenged mice plus two standard deviations. C Associations with protection were obtained by categorising mice into two or three groups following challenge: protected or delayed (p/d) vs. no delay (nd), or protection (p) vs. delay (d) vs. no delay (nd). Numbers represent $p$ values $t$ tests (comparing two groups) or ANOVA with Bonferroni's multiple comparisons test (comparing three groups). Means are shown \pm SD.

of these findings is to warrant an extension of the existing RTS,S and $R 21^{17}$ vaccines to include the junctional epitope. It has been shown here that combining vaccines including (NANP) 6 and the junctional epitope ADGN-long enhances protective efficacy compared to vaccines containing either epitope alone. The efficacy of either vaccine was low, but the purpose of the present study was only to identify the potential of a novel epitope in comparison with a known target of neutralising antibodies, NANP. The exact limits of the optimal extension of ADGN-long could be determined pre-clinically, using a VLP-peptide platform as here.

RI-based Q $\beta$-peptide showed no efficacy, despite the presented epitopes being recognised by a potently neutralising antibody ${ }^{16}$, and despite the antibodies generated by $\mathrm{Q} \beta-\mathrm{RI}$ vaccines showing excellent recognition of full-length PvCSP, suggesting that if a vaccine targeting $\mathrm{Rl}$ could be made to work, it could have crossspecies efficacy. The best results obtained with Region I or II peptide-based vaccines have used longer versions than those used in this paper, but still only achieved $50-60 \%$ protection against a very low dose challenge (100 sporozoites) ${ }^{18}$, or reduced liver burden by $18 \%$, low compared to $98 \%$ reduction obtained with a repeat-region based vaccine in the same study ${ }^{19}$. The epitope is cryptic, not being recognised when presented within the full-length $\mathrm{CSP}^{20}$, but inconsistently, so, being elicited in some volunteers by vaccination with radiation attenuated sporozoites $^{14,15}$. Separate vaccination with the $\mathrm{N}$-terminal region may succeed in eliciting neutralising antibodies, but that strategy would probably increase the expense of the vaccine if combined with, say, RTS,S or R21. A single-component vaccine consisting of the repeat region and the $\mathrm{N}$-terminal domain only, and missing the C-terminal domain, might allow neutralising antibodies to be generated against Region I as then CSP will be in a potentially more immunogenic conformation ${ }^{21}$.

The $Q \beta$-peptide platform was also used to uncover an apparently linear relationship between the number of NANP repeats and antiNANP titres. It has previously been shown that a PfCSP vaccine with 19 NANP repeats was more immunogenic and protective than one with 5 repeats $^{22}$. This was shown again here with short NANP peptides on $Q \beta$ with 1, 2, 3 and 6 NANP repeats, which showed increasing immunogenicity and protective efficacy with the number of repeats. The apparently linear relationship has not previously been observed, for no experiment has used more than two alternate NANP repeat numbers. The mathematical model here-in has presented, relating titre to peptide density, number of unit repeats per peptide, and dose, predicts a saturating effect, with extra NANP repeats ultimately failing to significantly increase titres. Increased density of NANP on a VLP has previously been associated with improved immunogenicity (675) and indeed antigen density also appears to dictate immune responses to blood antigens (676) The model accurately fits data from R21 and RTS,S varying dose and density (Florian Brod, personal communication) but it remains to be seen whether it also holds true when the number of unit repeats is increased. Potentially confounding this is evidence (Fig. 4E) that, at least at low numbers of (NANP) repeats, conformation varies; an assumption of the model is that this does not happen. If the model holds for NANP repeats, then the main benefit of longer NANP sequences presented at higher densities on VLPs will be dosesparing, helping reduce the cost of the vaccine, and hence increase its cost-effectiveness.

If short-peptide VLPs displaying malaria B-cell epitopes are to play some role in future vaccine development, it would be useful to know how to optimise immune responses against them. Malarial helper T-cell epitopes, or those from unrelated species such as the universal T-cell epitope from tetanus toxin, P2, have been used with most MAPs displaying CSP repeat peptides ${ }^{23-33}$, because without T-cell help the immune responses are very weak or absent ${ }^{24,25}$. With the $\mathrm{Q} \beta$-peptide vaccines used in this thesis, this is evidently not an issue, as high titres were always achieved against the peptide presented on the VLP. Nevertheless, a fusion of the P2 epitope to (NANP) $)_{3}$ for presentation on $\mathrm{Q} \beta$ enhanced titres, though only slightly and the improvement was not statistically significant. The affinity of anti-NANP titres was, however, significantly improved if P2 was present, which could be of value because this paper also presented data to show that affinity determines challenge outcome when anti-NANP titres are equal. Further studies could look at the effects on the immunogenicity of other T-cell epitopes, and warrants exploring the addition of non-native T-cell epitopes to existing PfCSP VLPs such as RTS,S and R21.

$\mathrm{Q} \beta$ can elicit $\mathrm{CD} 8^{+} \mathrm{T}$-cell responses in addition to $\mathrm{CD}^{+}$and antibody responses, but only at much higher doses than used here $^{34}$. This platform could therefore also be used to explore CD8 ${ }^{+}$ T-cell responses in future. As total lgG has been found to correlate better than any given IgG subtype with protection in this model (authors, unpublished data), the contribution to the protection of particular IgG subtypes was not explored here. However, it would be of interest to further examine the mechanism of protection induced by these vaccines by, for instance, cleaving the Fc region of elicited antibodies prior to sporozoite neutralisation assays to 
A

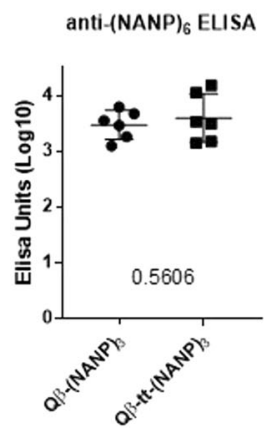

B

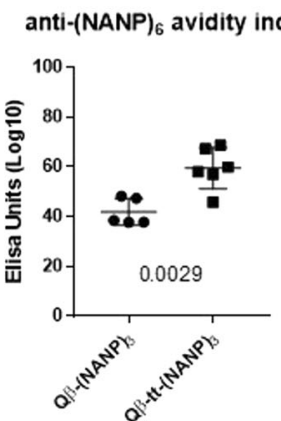

$c$

Survival: NANP vs ttNANP

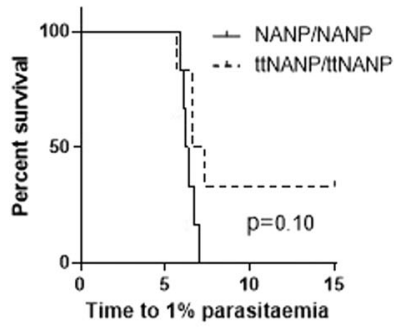

Fig. 6 Effects on immunogenicity and protective efficacy of tetanus toxin $\mathrm{CD}^{+}{ }^{+} \mathrm{T}$-cell epitope fusion with $\mathrm{Q} \beta$-(NANP) $)_{3}$ Q $\beta \beta$ VLPs were chemically coupled with tetanus toxin P2 epitope fused to (NANP) $)_{3}(\mathrm{Q} \beta \text {-tt-(NANP) })_{3}$ ) or just to (NANP) $)_{3}(\mathrm{Q} \beta \text {-(NANP) })_{3}$ ) and used to vaccinate $\mathrm{BALB} / \mathrm{c}$ mice ( $n=6$ per group) by intramuscular injection, using $1 \mu \mathrm{g}$ per dose, two shots, a 3 week prime-boost interval, with Matrix-M ${ }^{\mathrm{TM}}$ adjuvant. A Serum was collected from mice 2 weeks post-boost and standard curve ELISAs performed against (NANP) 6 . B The avidity index was calculated by taking the ratio of anti-(NANP) $)_{6}$ titres with and without treatment of sera with $7 \mathrm{M}$ urea. Numbers on graphs represent $p$ values from $t$ tests between groups. C Mice were challenged intravenously with 1000 PfCSP transgenic $P$. berghei sporozoites 3 weeks postboost. Daily thin blood smears were used to determine the time to reach $1 \%$ blood-stage parasitaemia by linear regression. $p$ Value from logrank test. Means are shown \pm SD.

determine whether there are Fc-mediated protective mechanisms, or whether protection is mediated entirely by blocking hepatocyte binding and entry. The persistence of antibody titres was not measured in this study, as the platform is not being developed for clinical use but rather as a tractable system for screening and exploring basic questions about the protective efficacy of epitopes. It would be of interest, however, to examine the longevity in this system, as another anti-CSP VLP platform, Rv21, was previously shown to elicit antibodies persisting at very high levels for over 9 months ${ }^{35}$.

In summary, the results here reported establishing that VLPpeptide vaccines are a tractable and inexpensive platform for conducting pre-clinical work important to informing clinical vaccine development.

\section{METHODS}

\section{Vaccination}

Vaccinations were performed by intramuscular injection using a $25 \mathrm{G}$ needle of $25 \mu \mathrm{L}$ vaccine formulation each into the right and left hind leg muscles (unless otherwise stated) of isofluorane-anaesthetised mice. Vaccines were administered at ranges from 1 to $20 \mu \mathrm{g}$, typically with two to three vaccinations administered at 3-week intervals unless otherwise stated. Matrix-M $\mathrm{M}^{\mathrm{Tm}}$ adjuvant (Novavax $\mathrm{AB}$, Uppsala, Sweden) was used at $12 \mu \mathrm{g}$ per dose. Adjuvants were kindly provided by Dr. Anita Milicic from the Jenner Institute adjuvant bank.

\section{Mouse strains used}

Six-week-old female BALB/C $\left(H-2^{d}\right)$ mice were used for vaccination experiments, with age-matched controls. To outbred mice and BALB/C mice were used for parasite maintenance and mosquito feeds. All mice from Harlan/Envigo.

\section{Ethics statement}

All animals and procedures were used in accordance with the terms of the United Kingdom Home Office Animals Act Project License. The procedures were approved by the University of Oxford Animal Care and Ethical Review Committee (PPL 30/2889 and P9804B4F1).

\section{Infection of Anopheles stephensi mosquitoes with $\boldsymbol{P}$. berghei}

Cryopreserved mouse blood stocks of wild type or transgenic $P$. berghei from liquid nitrogen were defrosted and immediately administered to naïve BALB/c or TO mice by intraperitoneal injection ( $100 \mu \mathrm{L})$. Thin blood smears were taken daily and when gametocytes were observed mice were anaesthetised by intramuscular injection (Rompun/Ketaset) for mosquito feed. Mosquitoes starved for $2 \mathrm{~h}$ were allowed to feed for $10-15 \mathrm{~m}$ on anaesthetised infected mice. Blood was taken from mice to confirm exflaggelation of gametocytes by microscopy. After feeding, mosquitoes were returned to fructose/P-amino benzoic acid on cotton wool and maintained in the Jenner Institute insectary $\left(19-21^{\circ} \mathrm{C}, 12 \mathrm{~h}\right.$ light/dark cycle). One week after feeding a second feed was performed on an anaesthetised naive mouse to improve sporozoite yields. Mosquitoes were maintained for a total of 21 days prior to dissection of sporozoite-infected salivary glands.

\section{Dissection of mosquito salivary glands and challenge of mice with sporozoites}

Twenty-one days after feeding on $P$. berghei infected mice, mosquitoes were sedated at $4{ }^{\circ} \mathrm{C}$ for dissection. Salivary glands were dissected from mosquitoes under a microscope and removed by pipette into a glass tissue homogeniser containing $100 \mu \mathrm{L}$ Schneider's insect media with $10 \%$ fetal bovine serum. Sporozoites were liberated from salivary glands by gently homogenising three times and counted using a haemocytometer. Sporozoite concentration was adjusted to $10^{4}$ sporozoites $/ \mathrm{mL}$ for intravenous injection into the tail vein of mice of $100 \mu \mathrm{L}(1000$ sporozoites per dose, by insulin syringe).

\section{Thin blood smears and calculation of time to reach $1 \%$ blood- stage parasitaemia}

Daily thin blood smears were prepared on glass slides from a drop of blood taken from the tail tip of challenged mice. Slides were fixed in methanol then stained in 5\% Giemsa (Sigma) for $30 \mathrm{~min}$ and washed in water. 1000 red blood cells were counted for three to five consecutive days until the mouse reached $1 \%$ blood-stage parasitaemia. Time to reach $1 \%$ blood-stage parasitaemia was calculated by linear regression of $\log _{10}$ percentage parasitaemia against time post-challenge, as described in ${ }^{11}$. Mice without parasites by day 15 were considered to have been conferred sterile protection against challenge. All data from all experiments carried out in this study are provided in the manuscript. Epitopes not showing any evidence of protective efficacy after one challenge experiment with 6 mice were not further pursued. All epitopes demonstrating evidence of protective efficacy were tested in at least two independent challenge experiments.

\section{Production of transgenic $\boldsymbol{P}$. berghei parasites}

$P$. berghei parasites expressing full-length PfCSP 3D7 (as shown in Fig. 2A) in place of PbCSP were produced by inserting an additional copy of the PfCSP gene at the $230 \mathrm{p}$ locus, under the control of the P. berghei UIS4 promoter, using the gene insertion/marker out approach ${ }^{17}$.

\section{$Q \beta$ VLP production, purification and chemical coupling}

Q $\beta$-transformed Escherichia coli from glycerol stock was grown to $1 \mathrm{~mL}$ in $\mathrm{LB} /$ carbenicillin, then transferred to $1 \mathrm{~L} \mathrm{M9}$ media (with $2 \mathrm{~mL} \mathrm{MgSO}, 5 \mathrm{~mL}$ $40 \%$ glucose, $50 \mathrm{~mL}$ casamino acid, $500 \mu \mathrm{L}$ vitamin B1 and $100 \mathrm{mg} / \mathrm{mL}$ 
Table 1. Peptides coupled to $\mathrm{Q} \beta$.

\begin{tabular}{ll}
\hline Name & Sequence \\
\hline (NANP) & \\
ttNANP & CGGNANPNANPNANP \\
$(\text { NANP })_{1}$ & CGGQYIKANSKFIGITENANPNANPNANP \\
$(\text { GGGG })_{2}(\text { NANP })_{1}$ & CGGNANP \\
$(\text { NANP })_{2}$ & CGGGGGGGGGNANP \\
$(\text { NANP })_{6}$ & CGGNANPNANP \\
Rl short & CGGNANPNANPNANPNANPNANPNANP \\
RI long & CGGEDNEKLRKPKH \\
ADGN long & CGGDDGNNEDNEKLRKPKHKKLKQPADGN \\
(NVDP $)_{6}$ & ADGNPDPNANPNVDPGGC \\
& CGGNVDPNVDPNVDPNVDPNVDPNVDP \\
\hline
\end{tabular}

carbenicillin) and incubated at $37^{\circ} \mathrm{C}$ for $18 \mathrm{~h}$. Cells were pelleted $(5000 \mathrm{~g}$, $25 \mathrm{~min}, 4^{\circ} \mathrm{C}$ ) and the supernatant discarded. The pellet was resuspended in PBS, centrifuged again $(20 \mathrm{~min}, 14,000 \mathrm{~g})$, and the supernatant discarded. The pellet was lysed using lysis buffer $\left(20 \mathrm{mM} \mathrm{NaPO}_{4} \mathrm{pH} 7.5,0.1 \%\right.$ triton $\mathrm{x}$ $100,5 \mathrm{mM}$ EDTA, $100 \mathrm{U} / \mathrm{g}$ cells Benzonase, $10 \mu \mathrm{L} / \mathrm{g}$ cells Lysonase, $10 \mu \mathrm{L} / \mathrm{ml}$ protease inhibitor), and freeze/thawing the pellet in dry ice twice. Lysed cells were sonicated for $1 \mathrm{~min}$ ( $15 \mathrm{~s}$ on/30 s off, 30\% intensity), centrifuged at $14,000 \mathrm{~g}$ for $25 \mathrm{~min}$, and the supernatant collected and filtered. Fractogel purification was carried out using $20 \mathrm{mM} \mathrm{NaPO}_{4} \mathrm{pH} 7.2$ buffer with either $150 \mathrm{mM}$ or $1 \mathrm{M} \mathrm{NaCl}$, followed by size exclusion chromatography.

Coupling $Q \beta$ peptides were performed by derivatising $Q \beta$ with reactive groups using succinimidyl-6-[( $\beta$-maleimidopropionamido)hexanoate] (SMPH) at $10 \times$ molar excess SMPH $(1 \mathrm{~h}, 50 \mathrm{~g} \mathrm{RT})$, followed by three $1 \mathrm{~m}$ $100 \mathrm{kDa}$ spin filtrations with PBS (Amicon $0.5 \mathrm{~mL}$ ) to remove free SMPH. Peptides were synthesised with free cysteines rendering SATA derivation unnecessary. Peptides were incubated with SMPH-derivatised $Q \beta$ for $3 \mathrm{~h}$ $(50 \mathrm{~g}, \mathrm{RT})$ and $\mathrm{Q} \beta$-VLPs stored at $-20^{\circ} \mathrm{C}$. Q $\beta$-VLPs consist of 180 identical subunits with four binding sites each and the binding efficiency of peptides was consistently at $80 \%$ for vaccines produced in this study. In consequence, approximately 575 peptides are presented per $Q \beta-V L P^{13}$.

A list of peptides coupled to $Q \beta$ and used as $Q \beta$-peptide VLPs in this paper is given in Table 1. All peptides were synthesised by ThinkPeptides.

\section{ELISAs: standard curve, affinity and subclass}

Nunc Maxisorp 96-well plates (Sigma) were coated with antigen $(50 \mu l$, $1 \mu \mathrm{g} / \mathrm{mL}$ in PBS) and incubated overnight at room temperature (RT). Plates were washed 6 times with PBS/0.05\% Tween (PBS/T) (Sigma) and blocked for $1 \mathrm{~h}$ with $10 \%$ skimmed milk (Sigma) in PBS/T (100 $\mu \mathrm{L} /$ well). Microvette serum tubes (Sarstedt) were used to collect blood from the tail veins of mice and serum obtained by centrifugation $(45,000 \mathrm{~g}, 10 \mathrm{~min})$. Sera was typically diluted at 1:500 post-prime, 1:1000 post-second shot and 1:2000 post-third shot and applied to plates in triplicate after blocking ( $2 \mathrm{~h}$ RT incubation). Standard curves were prepared on each plate against an antigen of interest by serial dilution of standard sera obtained by a cardiac bleed from mice vaccinated with the specific antigen being tested in ELISA. Plates were washed as before and goat anti-mouse whole lgG alkaline phosphatase conjugate (Sigma) applied $(50 \mu \mathrm{l} / \mathrm{well}, 1: 5000$ in PBS/T, $1 \mathrm{~h}$ RT). Plates were washed as before and $1 \mathrm{mg} / \mathrm{mL}$ pNPP (Sigma) in diethanolamine buffer (Pierce) applied to the plates (100 $\mu \mathrm{l} /$ well) and allowed to develop with readings on a BioTech Microplate Reader taken at $14 \mathrm{~min}$ and $1 \mathrm{~h}$ at $405 \mathrm{~nm}$. Titres were expressed as arbitrary ELISA units (EU) relative to a standard curve.

To determine the avidity index, a replicate ELISA was performed identical to and simultaneously with the standard curve ELISA, except that after $2 \mathrm{~h}$ incubation with diluted sera, $100 \mu \mathrm{L} 7 \mathrm{M}$ urea (Sigma) was applied to each well for $10 \mathrm{~min}$ (excluding the standard curve). Plates were then washed and the ELISA completed as before. The avidity index is the ratio of urea-treated to untreated ELISA units ${ }^{35}$.

\section{Statistical tests used}

GraphPad Prism (MacOS v6) and Microsoft Excel were used for all statistical analyses performed. Student's $t$ test and ANOVA with Bonferroni's multiple comparisons test were used on parametric data comparing two or more groups respectively. Log-rank (Mantel-Cox) tests were used to determine significant differences between survival curves.

\section{Statistical definition of challenge outcomes}

Three sporozoite challenge outcomes used in this paper are defined as follows. Sterile protection, or "protection", represents a case where a mouse has no detectable blood-stage parasitaemia by fifteen days postinfection. A mouse is classified as "delayed" if the time to reach $1 \%$ bloodstage parasitaemia in that mouse is greater than the meantime to reach $1 \%$ blood-stage parasitaemia of naïve or control mice in that experiment plus two standard deviations. A mouse is "not delayed" if the time to reach $1 \%$ blood-stage parasitaemia is less than two standard deviations above the mean of naïve or control mice.

\section{Reporting summary}

Further information on experimental design is available in the Nature Research Reporting Summary linked to this paper.

\section{DATA AVAILABILITY}

The data supporting the findings of the study are available from the corresponding author upon request.

Received: 17 November 2020; Accepted: 12 February 2021; Published online: 01 April 2021

\section{REFERENCES}

1. World Health Organization (WHO). World Malaria Report 2016 (2016).

2. RTS,S Clinical Trials Partnership. Efficacy and safety of the RTS,S/AS01 malaria vaccine during 18 months after vaccination: a phase 3 randomized, controlled trial in children and young infants at 11 African sites. PLoS Med. 11, e1001685 (2014).

3. Penny, M. A. et al. Public health impact and cost-effectiveness of the RTS,S/ASO1 malaria vaccine: a systematic comparison of predictions from four mathematical models. Lancet 387, 367-375 (2016).

4. Herrington, D. A. et al. Safety and immunogenicity in man of a synthetic peptide malaria vaccine against Plasmodium falciparum sporozoites. Nature 328, 257-259 (1987).

5. Ballou, W. R. et al. Immunogenicity of synthetic peptides from circumsporozoite protein of Plasmodium falciparum. Science 228, 996-999 (1985).

6. Young, J. F. et al. Expression of Plasmodium falciparum circumsporozoite proteins in Escherichia coli for potential use in a human malaria vaccine. Science $\mathbf{2 2 8}$, 958-962 (1985).

7. Vreden, S. G., Verhave, J. P., Oettinger, T., Sauerwein, R. W. \& Meuwissen, J. H. Phase I clinical trial of a recombinant malaria vaccine consisting of the circumsporozoite repeat region of Plasmodium falciparum coupled to hepatitis B surface antigen. Am. J. Trop. Med. Hyg. 45, 533-538 (1991).

8. Gordon, D. M. et al. Safety, immunogenicity, and efficacy of a recombinantly produced Plasmodium falciparum circumsporozoite protein-hepatitis B surface antigen subunit vaccine. J. Infect. Dis. 171, 1576-1585 (1995).

9. Brune, K. D. et al. Plug-and-display: decoration of virus-like particles via isopeptide bonds for modular immunization. Sci. Rep. 6, 19234 (2016).

10. Bejon, P. et al. Calculation of liver-to-blood inocula, parasite growth rates, and preerythrocytic vaccine efficacy, from serial quantitative polymerase chain reaction studies of volunteers challenged with malaria sporozoites. J. Infect. Dis. 191, 619-626 (2005).

11. Reyes-Sandoval, A. et al. CD8 ${ }^{+} \mathrm{T}$ effector memory cells protect against liver-stage malaria. J. Immunol. 187, 1347-1357 (2011).

12. Atcheson, E., Bauza, K. \& Reyes-Sandoval, A. A probabilistic model of preerythrocytic malaria vaccine combination in mice. PLOS ONE 14, e0209028 (2019).

13. Maurer, P. et al. A therapeutic vaccine for nicotine dependence: preclinical efficacy, and Phase I safety and immunogenicity. Eur. J. Immunol. 35, 2031-2040 (2005).

14. Kisalu, N. K. et al. A human monoclonal antibody prevents malaria infection by targeting a new site of vulnerability on the parasite. Nat. Med. 24, 408-416 (2018).

15. Tan, J. et al. A public antibody lineage that potently inhibits malaria infection through dual binding to the circumsporozoite protein. Nat. Med. 24, 401-407 (2018).

16. Espinosa, D. A. et al. Proteolytic cleavage of the plasmodium falciparum circumsporozoite protein is a target of protective antibodies. J. Infect. Dis. 212, 1111-1119 (2015). 
17. Collins, K. A., Snaith, R., Cottingham, M. G., Gilbert, S. C. \& Hill, A. V. S. Enhancing protective immunity to malaria with a highly immunogenic virus-like particle vaccine. Sci. Rep. 7, 46621 (2017).

18. Bongfen, S. E. et al. The N-terminal domain of Plasmodium falciparum circumsporozoite protein represents a target of protective immunity. Vaccine 27, 328-335 (2009).

19. Whitacre, D. C. et al. $P$. falciparum and $P$. vivax epitope-focused VLPs elicit sterile immunity to blood stage infections. PLOS ONE 10, e0124856 (2015).

20. Rathore, D. et al. An immunologically cryptic epitope of Plasmodium falciparum circumsporozoite protein facilitates liver cell recognition and induces protective antibodies that block liver cell invasion. J. Biol. Chem. 280, 20524-20529 (2005).

21. Coppi, A. et al. The malaria circumsporozoite protein has two functional domains, each with distinct roles as sporozoites journey from mosquito to mammalian host. J. Exp. Med. 208, 341-356 (2011).

22. Porter, M. D. et al. Transgenic parasites stably expressing full-length Plasmodium falciparum circumsporozoite protein as a model for vaccine down-selection in mice using sterile protection as an endpoint. Clin. Vaccin. Immunol. 20, 803-810 (2013).

23. Tam, J. P. et al. Incorporation of $\mathrm{T}$ and $\mathrm{B}$ epitopes of the circumsporozoite protein in a chemically defined synthetic vaccine against malaria. J. Exp. Med. 171, 299-306 (1990).

24. Valmori, D., Pessi, A., Bianchi, E. \& Corradin, G. Use of human universally antigenic tetanus toxin T cell epitopes as carriers for human vaccination. J. Immunol. 149, 717-721 (1992).

25. Herrera, M. A., de Plata, C., González, J. M., Corradin, G. \& Herrera, S. Immunogenicity of multiple antigen peptides containing Plasmodium vivax $\mathrm{CS}$ epitopes in BALB/c mice. Mem. Inst. Oswaldo Cruz 89, 71-76 (1994).

26. Wang, R. et al. Induction of protective polyclonal antibodies by immunization with a Plasmodium yoelii circumsporozoite protein multiple antigen peptide vaccine. J. Immunol. 154, 2784-2793 (1995).

27. Collins, W. E. et al. Protective immunity induced in squirrel monkeys with a multiple antigen construct against the circumsporozoite protein of Plasmodium vivax. Am. Am. J. Trop. Med. Hyg. 56, 200-210 (1997).

28. Yang, C. et al. Induction of protective antibodies in Saimiri monkeys by immunization with a multiple antigen construct (MAC) containing the Plasmodium vivax circumsporozoite protein repeat region and a universal T helper epitope of tetanus toxin. Vaccine 15, 377-386 (1997).

29. Moreno, C. A. et al. Preclinical evaluation of a synthetic Plasmodium falciparum MAP malaria vaccine in Aotus monkeys and mice. Vaccine 18, 89-99 (1999).

30. Nardin, E. H. et al. Synthetic malaria peptide vaccine elicits high levels of antibodies in vaccinees of defined HLA genotypes. J. Infect. Dis. 182, 1486-1496 (2000).

31. Nardin, E. H. et al. A totally synthetic polyoxime malaria vaccine containing Plasmodium falciparum $B$ cell and universal $T$ cell epitopes elicits immune responses in volunteers of diverse HLA types. J. Immunol. 166, 481-489 (2001).

32. Mahajan, B. et al. Multiple antigen peptide vaccines against Plasmodium falciparum malaria. Infect. Immun. 78, 4613-4624 (2010).

33. Gomes, A. C. et al. Adjusted particle size eliminates the need of linkage of antigen and adjuvants for appropriated $\mathrm{T}$ cell responses in virus-like particle-based vaccines. Front. Immunol. 8, 226 (2017).

34. Atcheson, E. et al. Tailoring a Plasmodium vivax vaccine to enhance efficacy through a combination of a CSP virus-like particle and TRAP viral vectors. Infect. Immun. 86, e00114-18 (2018). (9).
35. Grangeot-Keros, L. et al. Value of cytomegalovirus (CMV) IgG avidity index for the diagnosis of primary CMV infection in pregnant women. J. Infect. Dis. 175 944-946 (1997).

\section{ACKNOWLEDGEMENTS}

We would like to thank the Jenner Insectary for supplying infected mosquitoes for the studies. We are also grateful to Sarah Zakutansky, Alex Fyfe, Marta Ulaszewska, Sumi Biswas, and Alex Spencer for assisting with the production of transgenic $P$. berghei parasites in the Jenner Institute insectary. We thank Aadil el-Turabi, Ariane Cruz Gomez and Martin Bachmann for assistance with the production and supply of $\mathrm{Q} \beta$. The work was funded by a Wellcome Trust Career Development Fellowship award, Grant no. 097395/Z/11/Z, to A.R.-S., who is also a Jenner Investigator and an Oxford Martin Fellow. E.A. was funded by the Medical Research Council.

\section{AUTHOR CONTRIBUTIONS}

Experimental design: E.A., A.V.S.H., and A.R. Carried out experiments: E.A. Wrote the paper: E.A. Editing and paper revision: E.A., A.V.S.H., and A.R. Funding: E.A. and A.R.

\section{COMPETING INTERESTS}

The authors declare no competing interests.

\section{ADDITIONAL INFORMATION}

Supplementary information The online version contains supplementary material available at https://doi.org/10.1038/s41541-021-00302-x.

Correspondence and requests for materials should be addressed to A.R-S.

Reprints and permission information is available at http://www.nature.com/ reprints

Publisher's note Springer Nature remains neutral with regard to jurisdictional claims in published maps and institutional affiliations.

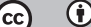

Open Access This article is licensed under a Creative Commons Attribution 4.0 International License, which permits use, sharing, adaptation, distribution and reproduction in any medium or format, as long as you give appropriate credit to the original author(s) and the source, provide a link to the Creative Commons license, and indicate if changes were made. The images or other third party material in this article are included in the article's Creative Commons license, unless indicated otherwise in a credit line to the material. If material is not included in the article's Creative Commons license and your intended use is not permitted by statutory regulation or exceeds the permitted use, you will need to obtain permission directly from the copyright holder. To view a copy of this license, visit http://creativecommons. org/licenses/by/4.0/.

(c) The Author(s) 2021 